\section{Representaciones culturales e identitarias en cambio: habitus científico y políticas públicas en ciencia y tecnología en la Argentina}

Adriana Stagnaro *

\begin{abstract}
Resumen: El trabajo presenta la interpretación del resultado de la yuxtaposición de dos dimensiones analíticas. Una genética, que abre una interrogación respecto de las representaciones culturales e identitarias de los integrantes del universo científico y tecnológico vigentes en el período neoliberal de la década de los 90', previo al cambio operado en la Argentina, desde el año 2003 en lo referente a las políticas públicas en ciencia y tecnología. La otra dimensión de orden antropológico se orienta al registro y diagnosis de las alteraciones y diferencias manifestadas en aquellas representaciones según los ensamblados sociales donde hayan emergido en tal período. Siempre a partir de un análisis etnográfico que toma por tópico y dispositivo al habitus científico y sus diversas manifestaciones (Bourdieu 2001, 2003; Wacquant, 2009). Se utilizarán al efecto, estudios realizados en comunidades científicas de biólogos y biotecnólogos, como así también en empresas mixtas y privadas consagradas a la producción biotecnológica.
\end{abstract}

Palabras clave: científicos - habitus -políticas públicas - representaciones culturales.

[Resúmenes en inglés y portugués en las páginas 325-326]

${ }^{(*)}$ Doctora de la UBA, con orientación en Antropología. Profesora de la cátedra de Epistemología y Métodos de la Investigación Social en la Carrera de Ciencias Antropológicas de la Facultad de Filosofía y Letras de la Universidad de Buenos Aires. Como investigadora del Instituto de Ciencias Antropológicas de esa institución fue una de las iniciadoras de la Antropología de la Ciencia y la Tecnología, especialidad sobre la cual dictó cursos de grado y posgrado en diversas universidades nacionales y extranjeras.

En el marco de los desarrollos actuales de la antropología de la ciencia, este trabajo se basa en el estudio de diversas configuraciones en que se expresó la cultura científica y tecnológica en la Argentina en la década de los años 1990. Dicho estudio consistió en una investigación etnográfica de larga duración de tres laboratorios de producción biotecnológica sitos en diferentes contextos locales de la República Argentina en los que se manifiestan distintas maneras de pensar, organizar, practicar y experimentar la vida científica de laboratorio. Cada uno de estas investigaciones se presentó como un caso diferenciado de la dinámica de conformación de las prácticas y discursos científicos. Su contraste y comparación dio cuenta de las distintas lógicas que animan e intervienen en la delimitación y disímil en- 
samblado de la experiencia en tales campos científicos académicos y empresariales, como así también de la compleja interrelación entre las prácticas teóricas, técnicas y sociales de los investigadores en los laboratorios argentinos de biociencias.

El modelo de Bourdieu (1991, [1984] 2008, [2001] 2003) nos proveyó los elementos necesarios para hacer explícitos todos o a la mayor parte de los condicionantes de la práctica social en los espacios de producción científica, como lo pueden ser el rol de las configuraciones sociales, de las instituciones y de las disciplinas en la determinación de las trayectorias y posiciones de los actores en el campo científico. Su teoría del mundo social nos brinda los conceptos de campo y habitus indisolublemente relacionados y operantes en las experiencias incorporadas en una trayectoria individual siempre dentro de un espacio social y dentro de un campo relativamente autónomo.

La búsqueda de ajustes estructurales provenientes no del laboratorio ni de los investigadores, sino por el contrario del espacio científico donde están inmersos, permitió indagar y develar sus lógicas genéricas y específicas, sin dejar de comprender que los científicos, los grupos de investigación y el laboratorio, según el tipo y el peso del capital científico que posean, determinan a su vez el estado de las fuerzas dentro del campo.

Así, el aporte de nuestra etnografía de la ciencia a este modelo de Bourdieu fue haber reconocido y examinado en la observación de las prácticas científicas y tecnológicas, distintos tipos de configuraciones o ensamblados (Rabinow, 2008) de elementos variados y disímiles, acompañados de la identificación de diversos tipos de habitus (Wacquant 2009), cuya presencia hace más compleja su comprensión, suspendiendo en ella el recurso a una más simple interpretación determinista derivada de una aplicación mecánica del modelo en cuestión. Siempre según nuestra perspectiva, otra de las contribuciones de nuestro trabajo es el haber mostrado que existen relaciones complejas entre los agentes, los objetos e instrumentos y los productos obtenidos por la actividad específica en cada uno de los casos examinados, y que los factores cognitivos, sociales, económicos, culturales y políticos en juego adquieren formas particulares en la configuración de cada uno de ellos, considerándolos en su especificidad y jerarquía, sin negar su actuación simultánea e interacción constantes en la dinámica científica. Es decir, constatamos que operan factores heterogéneos, pero no por ello imposibles de distinguir o diferenciar, y que el establecimiento de jerarquías de factores no es fijo ni establecido de antemano, sino van cambiando en virtud de la naturaleza variable de las situaciones.

El trabajo de campo se desarrolló en primer lugar en el laboratorio privado de una empresa biotecnológica de la Ciudad de Buenos Aires. Luego en otro laboratorio de características mixtas, bajo la forma societaria de empresa privada inserta en la estructura universitaria nacional de la Provincia de Santa Fe. Por fin, en un laboratorio de un instituto de investigaciones estatal sito en la Provincia de Tucumán. En estos tres momentos etnográficos sucesivos se observó un desplazamiento de la investigación desde la esfera privada de producción y aplicación científica de neto corte empresarial a un espacio social de tipo mixto, empresarial y universitario, para finalmente arribar a un campo institucional de carácter universitario estatal. Despliegue en concordancia con el devenir histórico y político general donde en la década de los años 90 se priorizaban y alentaban las lógicas privatistas del conocimiento científico, y que a partir del año 2003, se promovieron por el contrario, las restitutivas de políticas científicas estatales tendientes a la autonomía científica y al desarrollo social. 
La pesquisa desarrollada en Buenos Aires en una pequeña empresa biotecnológica dedicada a la investigación, desarrollo y fabricación de productos biotecnológicos para uso farmacéutico arrojó datos identitarios de sus fundadores, investigadores científicos provenientes del ámbito académico de la universidad pública. La idea primigenia que tenían los fundadores era dedicarse a las tareas de investigación y desarrollo de productos biotecnológicos, para después ofrecérselos a la industria farmacéutica para su comercialización y venta. Pero las circunstancias del contexto forzaron su objetivo hacia otras direcciones. Así pues, ante la ausencia de una demanda legítima por parte del sector industrial, se vieron obligados a asumir la comercialización. Este cambio se presentó como un desafío, consistente en afrontar todos los estadios hasta obtener un producto de uso como medicamento humano, con todos los requisitos de pureza y excelencia respecto de su calidad. También implicó conocer gran cantidad de disciplinas involucradas en este proceso: bioquímicas, químicas, farmacológicas y tecnológicas básicas de la industria. En este sentido, los actores se reconocieron como mediadores entre la ciencia pura y la ciencia aplicada, adscribiéndose a esta última en el momento de definir su quehacer, y como artífices de una interfase entre ellos. El posicionamiento en el campo de la ciencia aplicada les permitía demandar específicamente el conocimiento necesario al sistema científico, del cual se excluían, pero a su vez, la asunción de su formación científica y su participación en la cultura de la ciencia básica, los facultó para interpretar y traducir correctamente hacia la esfera tecnológica, construyendo así un espacio de interfase entre los dos sistemas. Mientras por un lado se definían por sus objetivos e intereses como tecnólogos, efectuando transferencia de tecnología, dominando múltiples técnicas y aplicándolas a su actividad de desarrollo y producción de productos farmacéuticos finales; por el otro destacaban la importancia de 'seguir haciendo ciencia', de mantener una conexión directa con ella. Este juego de oposición en sus posicionamientos autoriza a pensar que, aunque marquen una y otra vez las diferencias entre ciencia y tecnología, es su condición de científicos la que los habilita para transitar con éxito por un terreno algo ambiguo que definen como interfase.

No obstante, la adscripción consciente de los integrantes del laboratorio al mundo de la empresa biotecnológica y por ende al proceso de aplicación del conocimiento científico, redunda en una demarcación neta y en una extrañeza manifestada por sus miembros respecto de la esfera científica. Una vez traspasado con éxito el profundo abismo existente entre el suelo científico y el suelo empresarial, desde el lugar de empresarios, se ven impelidos a tomar distancia de su habitus científico para ejercer o aparentar dominar la aplicación y posterior comercialización de los productos biotecnológicos. En su contradictorio y urgido aprendizaje del oficio de empresarios, ponen en juego constantemente su capital de conocimientos científicos, ya sea para controlar internamente los desarrollos científicos y tecnológicos realizados en la empresa, para actuar como mediadores entre ésta y la universidad, o para reclamar desde la posición de innovadores, fondos públicos destinados a la creación tecnocientífica.

El contexto local de producción, bajo la influencia del modelo neoliberal que comenzaba a imponerse en la Argentina, es el que propendió a la intensificación de las relaciones entre la ciencia y la industria e impulsó a los científicos a transformarse en empresarios. En consecuencia la propia universidad promueve la creación de estructuras de articulación y acercamiento y pone en vigencia un encuadre jurídico y reglamentario ya anticipado 
en los países industrializados, pero sin la existencia en nuestro caso de un polo industrial que demande ciencia y tecnología. Por ello, los investigadores emprendedores, además de salir de las instituciones universitarias y de investigación para lanzarse al incierto mundo empresarial e industrial privado, deberán asumir todos los pasos productivos, desde el desarrollo 'puro' hasta la comercialización del producto final, o en su lenguaje, hasta obtener el 'frasquito final'. Tomar esa responsabilidad bajo las presiones de las demandas e imposiciones del campo económico, los acercó al modelo de la industria y en virtud de sus nuevos conocimientos relativos al 'saber hacer' se emplazan en un lugar de mediación, por su capacidad de traducir un lenguaje en otro. Ello a su turno, los coloca en una posición de poder, pues saben actualizar y valorizar sus conocimientos científicos de modo de transformarlos en productos industriales de demanda social.

Este tipo de ensamblado empresarial, representa el punto culminante de la transformación extrema de la producción científica hacia un modelo industrial, con la característica del sometimiento total a los intereses comerciales y la implicación también total de los científicos, ahora empresarios, en el proyecto empresarial y sus lógicas. La tensión resultante de la combinación de las prácticas científicas y comerciales se resuelve transformando el proyecto de científico aplicado a empresarial, y del propio científico a empresario. Este salto abismal desde la ciencia aplicada hacia la empresa, si bien muy innovador y receptor de las nuevas políticas científicas, tendientes a generar profundas mutaciones en el sistema nacional de investigación, también expresa cierta rigidez en resolver la compatibilidad de las dos lógicas -la científica y la comercial-, al exponer de pleno a los investigadores a las presiones y reglas propias del campo empresarial, donde tienen la desventaja de ser aventureros recién llegados y en el mejor de los casos, 'fundadores' adelantados.

Por otra parte se observa en los actores de este escenario social, un desplazamiento desde un habitus científico de origen hacia un habitus empresarial de destino, donde se hacen presentes todas las metamorfosis necesarias para adquirir las posiciones y disposiciones de éste último, que no se limitan a los cambios operados en la dimensión institucional, sino que alcanzan una profunda transformación psicológica de los agentes al momento de dar cuenta de sus prácticas mercantiles actuales. La mercantilización de los investigadores analizados exige una más íntima mutación subjetiva como condición de la internalización de la racionalidad económica propia del empresario de la ciencia. Su sistema de disposiciones y valores prioriza el interés económico por sobre el interés científico, marcando la discontinuidad entre actividad científica y oficio de empresario, no obstante lo cual no dejan de valorizar la importancia científica de sus trabajos en el pasaje hacia la empresa. $\mathrm{Al}$ involucrarse en el proyecto empresarial más como empresarios que como científicos, se proyectan con curiosidad en terra incognita, alejándose del mundo universitario y académico de formación para adentrase en uno más amplio y complejo como lo es el económico. Sin embargo no pierden de vista a la investigación académica, "es importante seguir teniendo una pata en la ciencia", tenida como fuente de conocimientos y destrezas específicas susceptibles de ser puestas en valor en el mercado y que constituyen la garantía del control industrial.

El segundo momento etnográfico estuvo marcado por el desplazamiento hacia otro ambiente tecnocientífico, el de una empresa incubada dentro de la universidad de la Provincia 
de Santa Fe. Dentro de ese contexto tuvimos la oportunidad de atender y registrar la emergencia de una nueva configuración de investigación biotecnológica cimentada en la puesta a punto y desarrollo de un proyecto innovativo, propuesto por un científico argentino con estudios de posdoctorado en Europa, en su búsqueda de reinserción en su país. Ésta finalmente se produce en un instituto santafesino que había establecido precedentemente lazos cooperativos con un instituto alemán de biotecnología. Por otro lado el proceso de incubación no estuvo exento de características agonísticas, manifestadas en las luchas y los conflictos que sostuvieron nuestro interlocutor a la sazón nuevo director del Instituto, con el director precedente y también con las autoridades de la universidad, quienes lo rechazaban, ofreciéndole serias resistencias a la implantación y desarrollo de su proyecto. Así, ante las estrategias de ingreso y posicionamiento en el campo,-participación en la presentación de proyectos, en concursos de cargos, en la nueva carrera de biotecnología, administración de fondos e interés científico en el desarrollo de su propio proyecto-, se opusieron en cada una de sus instancias acciones de impugnación por parte de los actores científicos y burocráticos ya instalados y posicionados en él en forma dominante. Ello impulsó a delinear estrategias de denuncia y crítica, interpretadas como 'subversivas' desde la lógica operante en el campo científico (Bourdieu, 1976) propias de los 'recién llegados' y antagónicas a las de 'conservación' correspondientes a los ya bien posicionados. Dentro del juego de fuerzas, rechazan el orden establecido. La discusión por parte del nuevo director de este orden y la crítica de las posiciones de los investigadores anteriormente instalados en el campo, como de sus carreras y trayectorias y autoridad científica, lo obliga a generar 'apuestas' más costosas y arriesgadas, poniendo en juego su capital científico, invirtiendo sus recursos y poderes sin esperar beneficios importantes a corto plazo.

Por otra parte, el director y su grupo se ven impelidos a participar de la lógica del campo universitario, por sus intereses en integrarse a su orden institucional como docentes concursados, investigadores legitimados e innovadores reconocidos para cumplir con la función específica de desarrollar sus prácticas científico-tecnológicas y producir conocimientos. En la liza para lograr una posición en este campo y el consecuente reconocimiento de ella, muestran el capital científico acumulado en sus trayectorias y exhiben asimismo una conducta pautada por el habitus correspondiente. El habitus universitario aquí señalado es considerado desde nuestra perspectiva como una forma especial del habitus académico, diferenciándose ambos del científico. Como principio organizador de las prácticas universitarias, refiere a 'potencialidades inscriptas en el cuerpo de los agentes' moldeado constantemente por los efectos de la estructura objetiva del mundo social, a las posiciones sociales o lugares adquiridos y a las tomas de posición o 'elecciones' realizadas por los agentes sociales en los diferentes ámbitos de la práctica.

Los científicos investigados debieron armar para sí mismos un habitus empresarial-como hicieron primeramente con el habitus académico-, porque estaban distantes de poseerlo. El know how de administración de empresas les era ajeno y desde el punto de vista estratégico, el único conocimiento que poseían era la existencia en ese momento en la Argentina de un área de vacancia en la producción de sustancias utilizadas como medicamentos para la salud humana, con el uso de una tecnología biológica totalmente novedosa. A partir de este punto, vislumbraron la forma de consolidar el capital simbólico reunido en sus tra- 
yectorias profesionales y su encarnación en un proyecto de vida y trabajo. Como primer objetivo se impuso la organización de la empresa y la obtención de recursos materiales para el equipamiento del laboratorio.

En la reflexión respecto a esas tareas, reconocían como aspecto positivo el espacio de libertad conseguido. En oposición a las representaciones más arraigadas respecto de la ciencia aplicada llevada a cabo en espacios privados, que remarcan el carácter rutinario y apremiante de la actividad, valorizaron el espacio empresarial al apreciar algunos de sus rasgos de independencia e inmunidad frente a la burocracia universitaria.

La valoración de la independencia y libertad otorgada por el espacio formal de la empresa, se conectaba con la posibilidad de disponer de los materiales y equipamiento, en cualquier locus que ocupe, ya sea universitario o no, y con la viabilidad de conseguir financiamiento privado en caso de tener que alejarse de las arenas universitarias, donde su posicionamiento estaba siempre cuestionado, a pesar del capital científico puesto en juego.

El grupo de investigación se distinguió del resto de los actores del campo universitario por sus vinculaciones con el exterior, en particular con centros de investigación de la entonces Comunidad Económica Europea, con quienes firmaron convenios de cooperación, transferencia y consolidación de proyectos que le permitió adquirir equipamiento de gran valor tanto para la universidad como también para su propia experimentación.

En el relato de su director encontramos no obstante, una apreciación contradictoria y paradojal respecto a la forma de percibir su actividad y la explicitación de sus metas. En él se trasluce la tensión propia de la dualidad del mundo científico donde conviven dos tipos de prácticas, la de investigación en la universidad y la de la demanda social, esfera del desinterés la primera y de la recompensa económica la segunda, a favor de la cual se hacen determinadas concesiones (Biagioli, 1988 apud Bourdieu, 2003, pp. 95-96).

Desde el locus universitario estableció estrechas conexiones con el mundo industrial, participando activamente en él a través de relaciones de cooperación y asociación con empresas biotecnológicas y hasta formando una de ellas dentro mismo del campo universitario, no obstante lo cual tampoco asume el rol de empresario. Su experiencia en ambas esferas de actividad, lo ubicaba en un punto liminar al que definía como interfase entre universidad y empresa.

Dicha posición liminar o fronteriza entre aquellos dos mundos era percibida por el actor como contradictoria. Por un lado, la participación en actividades de investigación, la creación de un grupo y la generación de un espacio de formación con orientación hacia la publicación. Por el otro, el desarrollo de actividades de científico-empresario con pragmatismo, teniendo en cuenta en sus proyectos, la posibilidad de 'vender una molécula' en el mercado a precios competitivos, de 'hacerla producir como una fábrica', posibilitando el acceso al dinero y la rentabilidad e impacto social de su quehacer. Estas dos caras del 'trabajo con ciencia' se complementan en la capacidad de competir, ya sea en la ciencia básica o aplicada. La forma de competencia es limitada y a diferencia de la planteada por Callon (1995) no es libre, por no haber un acceso amplio e indiferenciado al mercado científico internacional, motivado entre otras cosas en la imposibilidad de sostener el desarrollo de una 'ciencia de punta' altamente competitiva. Ante tal panorama, al científico local le queda como opción replegarse -ya desde sus tiempos iniciales de formación y enculturación, aconsejado por sus directores de beca, del laboratorio o instituto- a hacer una ciencia de 
'segunda línea', sabiendo de antemano que no podrá ganar la carrera darwiniana hacia la primera publicación, la 'primer foto' según la metáfora utilizada por el director. Su estrategia entonces, invita a quedarse en los márgenes de la investigación científica avanzada, donde encontrará un locus específico que le permita hacer una buena contribución, también original, susceptible de ser publicada, pero sin revestir el carácter de problemática interesante por su anticipo de novedad en la primera línea de investigación.

Las prácticas, discursos y valores relevados en la etnografía muestran que el término 'científico’ no sólo es descriptivo de una actividad, sino que constituye una categoría cultural cargada de significados complejos y contradictorios, susceptibles de ser utilizados e interpretados por los interlocutores en el contexto social de actuación.

Desde el marco de la teoría de las prácticas, que nos ha enseñado que los agentes sociales actúan en un espacio donde no se hallan totalmente libres ni totalmente constrictos, participando tanto en la reproducción como en la transformación de los sistemas culturales, las categorías culturales y las estructuras sociales no son esquemas rígidos o reglas a las cuales los actores sociales deban ajustarse en su vida cotidiana, sino que proveen recursos que aquéllos utilizan y manipulan en el plano de la acción y de la retórica.

En el caso bajo estudio, interpretamos que el actor principal, el director del laboratorio expresa una crisis acerca de su identidad social como científico, no pudiendo adscribirse íntegramente a la categoría cultural de científico o de investigador puro, ni a la de tecnólogo pragmático. Esta ambigüedad identitaria se refleja en sus definiciones de objetivos contrapuestos, en sus prácticas y en su sistema de valores, los que operan en campos sociales con lógicas diferentes: la universidad y la empresa biotecnológica. La multiplicidad y heterogeneidad de actividades, de disciplinas y conocimientos involucrados en esos campos, se entrelazan, intersecan y entran en conflicto, originando así dudas en la interpretación del contexto cooperativo de la actividad tecnocientífica. Las tensiones, entonces, originadas en esa ambigüedad, no tienden a resolverse en una sola dirección -o sea hacia un lado u otro de los campos-, sino que lo que se preserva es la contradicción. El juego cultural de la ambigüedad, permite al director ubicarse entre dos mundos y moverse con facilidad traspasando sus fronteras, traduciendo bilateralmente sus distintos lenguajes, lo que le da acceso a variados recursos de legitimidad, reteniendo la independencia y poder que resulta de ellos.

Esta posibilidad de actuar en los dos mundos, de viajar dentro y hacia fuera de ellos, lo constituye en un 'embajador de la ciencia' (Zabusky, 1992), actuando como 'guardián' de ella, lo que es su raison d'être, dentro de la plataforma tecnológica que supo armar y recrear. Para mantener viva la llama del interés por las preguntas básicas, debe negociar entre intereses de parte, en beneficio y para la preservación de un espacio de actividad pura y desinteresada, la que en su imaginario no debe desaparecer arrasada por la lógica pragmática. En el espacio liminar y fronterizo que ocupa entre mundos estructurados con lógicas opuestas crea ambigüedad en los intersticios de las estructuras, utilizándolas y explotándolas en sus prácticas, de modo consciente o inconsciente y expresándolas directamente (interfase) o indirectamente en sus expresiones discursivas.

Como personaje liminar siempre corre el riesgo de desaparecer, dada la dificultad atribuida a los tipos liminares de ser caracterizados y capturados en términos clasificatorios. $\mathrm{Al}$ no conseguir la contención del medio universitario local, busca el apoyo en la arena científica internacional y asimismo en la empresarial, protectoras ambas de su emprendimiento 
de investigación, desarrollo e innovación local, con el objetivo de consolidar la realización de sus proyectos.

A diferencia de los integrantes del primer laboratorio presentado-, quienes se excluyen de la comunidad científica definiéndose como empresarios ('no somos científicos, dejamos de hacer ciencia'), el director del segundo, se adscribe a la categoría cultural de científico a partir de la cual identifica 'hacer ciencia' con hacer ciencia básica, no se excluye de la comunidad académica, trabajando dentro de ella en ciencia básica y aplicada y generando transformaciones dentro de la esfera universitaria, al introducir formas de organización y valores propios de la industria. Resuelve así en parte las tensiones surgidas de la oposición academia-industria.

La iniciativa se inscribió en una época donde la política científica nacional alentó los procesos de valorización económica de los proyectos científicos innovadores, favoreciendo los modelos de privatización y mercantilización de la ciencia. Así se promulgaron en nuestro país leyes de innovación científico-tecnológica de rango nacional y provincial, organizándose de tal modo el armado de estructuras que incitaban a la vinculación entre las universidades y las empresas en ellas incubadas. Dentro de aquellas nuevas estructuras se dispusieron normas que favorecieron y beneficiaron los nuevos posicionamientos de los investigadores de vanguardia. El estatus de incubadores fue reconocido legalmente en este nuevo espacio, para lo cual se tuvo que facilitar que los profesores, docentes e investigadores públicos de las universidades o centros de investigación nacional o provincial, pudieran desempeñarse simultáneamente como directores de empresas creadas por ellos a fin de aumentar la importancia de los proyectos e investigaciones llevados a cabo en el ejercicio de sus funciones.

En este contexto, el director del laboratorio y creador de la empresa manifiesta un tipo de compromiso empresarial que a pesar del levantamiento de las vallas institucionales y a diferencia de los casos anteriores, se caracteriza por no invadir su identidad de investigador, quedando en consecuencia a resguardo su trayectoria científica. A partir de su posición particular interpreta las prácticas científicas y económicas haciendo tanto diferenciaciones como aproximaciones. De tal modo le concede a la distinción histórica entre 'ciencia básica' y 'ciencia aplicada' un efecto esquematizador y enculturizador, y a su vez irrelevante a la hora de hacer elecciones temáticas en el marco de la práctica científica local. En su lugar, lo que surge como determinante es la capacidad restringida de competir local o globalmente. Entonces, las categorías nativas de 'ciencia de punta' y ciencia de 'segunda línea', representativas de las prácticas científicas y tecnológicas resultantes de la división internacional del trabajo, vienen a sustituir las distinciones anteriores. Así resignifican desde el locus propio las lógicas cosmopolitas.

La investigación reveló como característica más destacada de la fundación de la empresa por parte del director e investigador principal del equipo universitario, la búsqueda preferente de beneficios de orden científico y tecnológico respecto de las utilidades y provechos comerciales, contrariamente a lo que indica el sentido común empresarial. En consecuencia se observa un bajo nivel de mercantilización del investigador fundador y de su grupo y un bajo compromiso empresarial. Su figura de investigador universitario y creador de la empresa, no coincide plenamente con ninguna de las representaciones extremas y contrapuestas del científico ligado a la búsqueda desinteresada de conocimiento, recluido en 
lo recóndito en su laboratorio universitario en resguardo de las normas y los valores de la comunidad científica frente a la penetración de las lógicas económicas -por una parte-, y la del científico perteneciente a la industria, abierto al mundo visible de la empresa y sujeto a sus condiciones - por la otra-.

Nuestro análisis muestra que la oportunidad de crear una empresa no surge directamente de un condicionamiento económico sino de la percepción de la conveniencia de esa creación como dispositivo favorable para el desarrollo del proyecto de investigación y la estabilidad y reproducción social del grupo de científicos. La explicación dada por el director es elocuente: 'generamos la empresa como herramienta para conseguir fondos'. Por ello es que el proyecto empresarial implícito depende para su coronación del valor científico de los resultados obtenidos y por consecuencia el interés mayor descansa en este último por encima del valor atribuido a la expansión económica.

La participación del equipo científico, y en especial su director, en la gesta y desarrollo de un proyecto empresarial dentro del campo universitario, ha sido beneficioso para el desenvolvimiento exitoso del proyecto científico y para el estilo de trabajo de investigación, en especial en el adelanto y refinamiento de su equipamiento (adquisición de un biofermentador de modelo inexistente aún en América Latina). Con esa base tecnológica las prácticas de laboratorio experimentaron grandes cambios, abriéndose nuevas vías investigativas, estimulando el planteo de nuevos problemas, intercambios y colaboraciones, ampliando en general el horizonte hacia nuevos perfiles innovativos y aumentando su visibilidad y reconocimiento. Desde otra perspectiva, la creación de la empresa facilitó asimismo la constitución, consolidación y reproducción social del grupo, el mantenimiento y soporte de las carreras y trayectorias, al generar autonomía y control en los recursos financieros, menoscabando las asfixias de financiación y remuneración impuestas por las políticas y gestiones universitarias.

En relación a la posibilidad de conformación de un habitus empresario, resultado de la creación de la empresa como contexto de actuación, podemos afirmar que en el caso de Santa Fe no se llegó a organizar como sistema de percepciones y disposiciones. Sí, fue de interés por parte del director demostrar al campo universitario su capacidad de forjar y reflejar una imagen de empresario de la ciencia y de espíritu de empresa, dirigida a los que la quieran ver, pero no absorbida interna ni culturalmente. Por ello exteriorizó una máscara empresarial para preservar los dominios donde ejercer su habitus científico y universitario, pero no el empresario. Este hallazgo nos da pie para criticar, en base a las características presentadas en nuestro ejemplo, a las posiciones que conciben a la ciencia, la tecnología, la industria y la empresa como esferas de actividad indiferenciadas en virtud de lo cual el proceso de mercantilización operado dentro de las instituciones científicas se disemina hasta llegar a impregnar las prácticas y disposiciones de los investigadores, quienes se transformarían sin renuencia en empresarios. Por el contrario el ejemplo demuestra que el artificio ideado para evitar tal mutación, tiene por objetivo 'venderse hacia el afuera como empresarios' para conservar y custodiar, en alguna medida y por algún tiempo, el fuego sagrado de la investigación científica. Al examinar en el presente caso los rasgos y matices de la transformación experimentada en la identidad de los investigadores, nos encontramos con la permanencia de un perfil de científico creador de empresas, débilmente embarcado en la aventura empresarial, y no obstante aún ligado y aferrado a la forma de 
vida académica y universitaria. El espíritu de empresa no es tan atractivo ni irrenunciable como las teorías de las nuevas lógicas científicas aspiran a presentarlo.

En el tercer caso bajo análisis, de un instituto de investigación estatal en la Provincia de Tucumán, la forma de coordinación de las prácticas científicas, tecnológicas y comerciales observadas manifestó un alto grado de heteronomización disciplinar y una intersección anticipada entre la esfera de investigación aplicada y su transferencia tecnológica de origen público, por un lado, y entre la esfera de producción comercial y su relación con la industria, por el otro. Su historia de transformaciones se inició desde un comienzo como 'cultura de producción' a gran escala de microorganismos en procesos industriales (planta piloto), para luego incorporar un área biotecnológica de desarrollo de los resultados de la investigación 'significativa' y su articulación con el sector industrial local nacional e internacional. Estos antecedentes, se unían a su amplia experiencia en vincularse con distintos tipos de instituciones públicas y empresas nacionales e internacionales de relevancia, lo que manifestaba a su vez una orientación firme hacia la valorización económica de su producción científica y tecnológica. De este modo los integrantes del instituto, si bien anclados en un ámbito académico y en un sistema de investigación pública, estaban familiarizados con las prácticas tecnológicas y productivas. Por ello, en tanto investigadores enculturados en tradiciones científicas e ingenieriles, no apelaban -como en los otros dos casos- a las categorías nativas distintivas entre 'ciencia pura, 'ciencia aplicada' y 'tecnología' para definiciones cognitivas de sus quehaceres, restándoles importancia en tal sentido. Tenían incorporadas las dos lógicas y desarrollaban sus prácticas en base a esa versatilidad adquirida, sin mayores deslices o conflictos.

El director del instituto, por ejemplo, resaltaba el perfil ingenieril del instituto para explicar el aporte al surgimiento de líneas de investigación heterogéneas, el desarrollo de capacidades científicas y profesionales involucradas con la mejora de los dispositivos experimentales y el 'control de calidad'. Asimismo el contacto con la industria, contribuyó positivamente a la valoración social y la regularidad en la ejecución de proyectos profesionales de investigadores, becarios y técnicos y a su tiempo de trabajo en el laboratorio. En cuanto a los proyectos científicos, la totalidad de los investigadores tenía una percepción positiva de los efectos de su implicación en el proyecto productivo respecto del trabajo de investigación, por la posibilidad de abrir líneas de investigación imprevistas, estimular otro tipo de pensamiento creativo y en especial poder disponer de equipamientos y dispositivos cada vez más actualizados. En general los científicos afirmaban obtener beneficios científicos y profesionales de su implicación con el sector productivo, traducido en un aumento del reconocimiento por parte de la comunidad científica al realizar transferencias tecnológicas de utilidad industrial y viceversa, por el retorno de beneficios económicos de la industria hacia el laboratorio, que se traducían en el mantenimiento y sostenimiento de distintos tipos de investigaciones.

Desde el punto de vista del director, el balance de la combinación de las actividades productivas y científicas era altamente positivo porque había, como hemos descripto, reciprocidad de beneficios de unas con las otras. Los roces e incompatibilidades que podían surgir eran minimizados en sus efectos, ante la capacidad de subsistencia y de reproducción social conferida por la producción industrial por fermentación a gran escala y los desarrollos en fisiología microbiana. 
La aproximación al estudio de los grupos de investigación dio lugar a la identificación de la categoría nativa de trabajo, que representa la vivencia generalizada de la forma de hacer ciencia en el laboratorio, las diferentes dinámicas adoptadas y la presencia fuerte de una división del trabajo interna y sistema de jerarquías en su interior. Apela asimismo a la valorización del quehacer científico con objetivos de satisfacción de necesidades locales, por lo cual 'hacer bien las cosas' ya no significaba someterse a las reglas de la ciencia básica o aplicada, en su caso, sino en desplazar las prioridades temáticas y objetivos impuestos desde lógicas externas del sistema internacional y atraer financiación hacia los temas relacionados a problemáticas regionales relevantes. Coincide con las categorías nativas utilizadas en Santa Fe de 'trabajar con ciencia' en nichos originales pero de 'segunda línea' y de abstenerse de competir en temas seleccionados por la comunidad internacional. La categoría amplía su extensión hasta llegar a abarcar la apreciación de que cuando en el trabajo científico o tecnológico se prioriza el interés y la inserción local, se supera la distinción entre ciencia básica y ciencia aplicada.

En la interpretación de la categoría 'trabajo' convocamos al concepto de sistema experimental (Rheinberger 1977) para dar cuenta de la interconexión entre los objetos científicos y sus condiciones técnicas y sociales de producción. Sólo es dentro de estos sistemas o unidades concurrentemente locales, individuales, sociales, institucionales, técnicas, instrumentales y epistémicas donde se genera una dinámica propia y específica, en la que los investigadores son reflexivos acerca de las características de sus objetos de indagación. En este contexto sus pensamientos y evaluaciones contemplan la reformulación de sus objetivos a fin de dar respuestas a las preguntas relevantes que emanan del ámbito local. En Tucumán se configuró un tipo de ensamblado heterogéneo y divergente de prácticas científicas con marcada tendencia aplicada hacia el sector productivo, de gran complejidad y diversificación en cuanto a objetivos e intereses, más afín a un tipo de cultura transversal que a una disciplinar o institucional, cuya práctica de investigación estaba moldeada por un habitus productivo. Dicho habitus motivaba a los investigadores de esa institución pública a participar y relacionarse con agentes del sector industrial, combinando dúctilmente rasgos académicos y empresariales en el contexto local.

En ese sentido comprobamos que el compromiso científico o tecnológico no los llevaba a jerarquizar ab initio sus trabajos en virtud de su importancia científica o económica, pudiendo desarrollar sus proyectos microbiológicos y biotecnológicos sin mayor contradicción, complementándose y confluyendo en ciertos puntos de unión. Articulaban así también los proyectos productivos con las empresas interesadas en un movimiento de acercamiento y distanciamiento entre las fronteras de la investigación y las de lo empresarial, reconociendo que estos eran campos con lógicas diferentes que era importante mantener separadas. No obstante lo cual estaban expuestos a las imposiciones de cada una de esas lógicas y a partir de ese punto optaban por una actitud flexible de avances y retrocesos sin intentar reducir la una a la otra, sino más bien producir una dinámica que contemplase a ambas y aflojara las tensiones a través de la expansión de orientaciones, capacidades y desempeños.

En la fluctuación entre la investigación científica y la vinculación con el sector empresarial se observó la libertad de los investigadores para declarar su vocación por la investigación sin subestimar la importancia de los factores económicos, y mostrar su talento para establecer contactos con el mundo empresarial sin llegar a incorporar un grado de mercanti- 
lización importante. La inclinación a hacer esos contactos no se basó en que confirieron una validez intrínseca a ese tipo de vinculación, sino más bien en la conveniencia de las colaboraciones puntuales con la industria para el aseguramiento de la reproducción social de los grupos de investigación existentes en el instituto y su mejoramiento profesional, a través del aumento de los recursos financieros y de los fondos de origen privado.

A diferencia de los empresarios científicos de Buenos Aires, los científicos de Tucumán no se sometieron a la autoridad irrestricta de las lógicas empresariales, sólo aceptaron seguir sus directivas en los períodos en que se desarrolló el contacto con la industria, teniendo en cuenta la eficacia de los modelos de colaboración. En contraste con el caso de Santa Fe, no necesitaron instrumentalizar la relación con empresas para asegurar fines científicos y proyectos individuales, ya que gozaron de posicionamientos profesionales más estables en su institución. Vistos en perspectiva, lucen como los más exitosos en lograr una relación equilibrada entre los aspectos científicos y comerciales de sus proyectos, sin poner en riesgo a ninguno de ellos, alternando momentos, estableciendo fronteras y definiendo su identidad a cada instante.

El estudio detallado de los casos presentados desde una perspectiva antropológica permitió recomponer las ondulaciones y recodos del itinerario científico y comercial de los proyectos implicados, y las fuerzas y movimientos de las diferentes dinámicas que animaron las prácticas concretas y el significado de las actuaciones de los sujetos en los distintos tipos de ensamblados identificados. Esta tarea tuvo como objetivo mostrar cómo bajo un mismo modo de restricción estructural, como lo fue la etapa neoliberal de la década de 1990 y sus efectos e implicaciones en las políticas y direccionamientos diseñados en el campo de la ciencia, la tecnología y la innovación, emergieron distintos tipos de configuraciones o ensamblados de conocimientos, prácticas de investigación y prácticas comerciales, que respondieron diferentemente al avance de las tendencias privatizadoras de la economía. En el campo científico dichas tendencias se expresaron por el impulso hacia la mercantilización de las prácticas públicas, privadas o mixtas de investigación.

En tal medida, dichos ensamblados expresan la complejidad científica, técnica y productiva de la investigación biotecnológica y de las interacciones entre el sector público de investigación y el privado de producción en el período analizado. Su estudio ayuda por otra parte a retratar los perfiles de carne y hueso del universo concreto, particular e idiosincrásico de la innovación científica actual en el país, ausentes en los abordajes macro analíticos económicos tenidos generalmente en cuenta a la hora del trazado de las políticas públicas en ciencia y tecnología.

Estando la Argentina inmersa en el presente sistema global de intercambios, que es a su vez un sistema global de desigualdades, la imagen de la ciencia y de la tecnología que sustenten las políticas públicas en ciencia, tecnología e innovación productiva jugará un rol crucial para determinar formas de acceso a los recursos, creación de riquezas y autonomía en el país. Confiamos en que el estudio antropológico, basado en el trabajo a escala personal con gran detalle de matices y particularizaciones, contribuya a una más profunda comprensión de las características especiales y el dinamismo de la investigación biotecnológica que se desarrolla en su seno. 


\section{Bibliografía}

Bourdieu, P. [2001] (2003). El oficio de científico. Ciencia de la ciencia y reflexividad. Barcelona: Anagrama.

[1994] (1997). Razones prácticas. Sobre la teoría de la acción. Barcelona: Anagrama.

(1991). El sentido práctico. Madrid: Taurus.

[1984] (2008). Homo academicus. Buenos Aires: Siglo XXI.

(1976). La specifité du champ scientifique et les conditions sociales du progres de la raison. En: Actes de la Recherche en Sciences Sociales No 2/3. 88-104.

Bourdieu, P. y Wacquant, L. J.D. [1992] (1995). RESPUESTAS. Por una antropología reflexiva. México: Grijalbo.

Callon, M. (1995). Four Models for the Dynamics of Science. En Handbook of Science and Technology Studies. Jasanoff, S., Markle, G., Petersen, J. y Pinch, T. Editores. London, Sage, 29-63.

Rabinow, P. (2008). Marking Time. On The Anthropology of the Contemporary. Princenton and Oxford: Princenton University Press.

Rheinberger, H. J. (1997). Toward a History of Epistemic Things. Stanford: Stanford University Press.

Wacquant, L. (2009). Habitus as topic and Tool: Reflexions on becoming a Fighter. En Shaffir, W.; Puddephatt, A. and Kleinknecht, S. (eds.) Ethnographies Revisited. New York: Routledge. 137-151.

Zabusky, S. (1992). Multiple Contexts, Multiple Meanings: Scientist in The European Pace Agency. En Hess, D. y Layne, L. eds. Knowledge and Society: The Anthropology of Science and Technology. Londres: JAI Press.115-142.

Summary: This essay introduces the interpretation of the yuxtaposition of two analitic dimensions. One of them of genetical bias, ask for cultural and identitary representations from members of scientific and technologic universe during decade 90'neoliberal period, previous at the change of public politics of science and technology operate since 2003 in Argentina. The other dimension of anthropological bias try to register and make the diagnosis of manifested alterations and differences in that representations in that period in accordance with social assemblages. Always from one ethnographic perspective in which scientific habitus and their manifestations are taken as topic and tool (Bourdieu, 2001, 2003; Wacquant, 2009). The utilized frame is the studies made in scientifics communities of biologyst and biotechnologist and in private and mixed biotech companies .

Keywords: cultural representations - habitus - public policies - scientists.

Resumo: $\mathrm{O}$ trabalho apresenta a interpretação do resultado da yuxtaposicão de duas dimensões analíticas. Uma genética, que abre uma interrogação com relação às representações culturais e identitárias dos integrantes do universo científico e tecnológico vigentes no período 
neoliberal da década dos '90, prévio às mudanças, prévio à mudança operada na Argentina, desde o ano 2003 naquilo referido às políticas públicas em ciência e tecnologia. A outra dimensão de ordem antropológica orienta-se ao registro e diagnose das alterações e diferenças manifestadas em aquelas representações segundo os ensamblados sociais onde emergiram nesse período. Sempre a partir de um análise etnográfico que toma por tema e dispositivo al habitus científico e suas diversas manifestações (Bourdieu 2001, 2003; Wacquant, 2009). Utilizar-se-ão estudos feitos em comunidades científicas de biólogos e biotecnólogos, como assim também em empresas mistas e privadas dedicadas à produção biotecnológica.

Palavras chave: científicos - habitus - políticas públicas - representações culturais. 